\title{
Temperature-Responsive Hydrophobic Silica Nanoparticle Ultrasound Contrast Agents Directed by Phospholipid Phase Behavior
}

\author{
Nicholas T. Blum ${ }^{1}$, Adem Yildirim ${ }^{1, \dagger}$, Ciara Gyorkos $^{1}$, Dennis Shi ${ }^{1,}$, Angela Cai ${ }^{1, \ddagger}$, Rajarshi $^{1}$ \\ Chattaraj $^{2,+}$, Andrew P. Goodwin ${ }^{1, *}$ \\ ${ }^{1}$ Department of Chemical and Biological Engineering, University of Colorado Boulder, Boulder, \\ CO 80303 USA
}

${ }^{2}$ Department of Mechanical Engineering, University of Colorado Boulder, Boulder, CO 80309 USA

\section{Abstract}

In this paper, we report ultrasonically-active nanoscale contrast agents that behave as thermometric sensors through phase change in their stabilizing phospholipid monolayer. Phospholipid-stabilized, hydrophobic mesoporous silica nanoparticles (P@hMSNs) are known to interact with high intensity focused ultrasound (HIFU) to promote cavitation at their surfaces, which can be used for both imaging and therapy. We show that the lateral lipid phase behavior of the phosphocholine lipid dictates the acoustic contrast of the P@hMSNs. When the lipids are in gel phase below their melting temperature, the P@hMSNs generate detectable microbubbles when exposed to HIFU. However, if the lipids exhibit a liquid expanded phase, the P@hMSNs cease to generate bubbles in response to HIFU insonation. We verify that the heating and subsequent transition of lipid coating the hMSN is associated with the loss of acoustic response by doping laurdan dye into the lipid monolayer and imaging lipid phase through red shifts in emission spectra. Similarly, cessation of cavitation was also induced by adding a fluidizing surfactant such as Triton X, which could be reversed upon washing away excess surfactant. Finally, by controlling for the partial fluidization caused by the adsorption of protein, P@hMSNs may be used as thermometric sensors of bulk fluid temperature. These findings not only impact the utilization of nanoscale agents as stimulusresponsive ultrasound contrast agents, but also have broader implications for how cavitation may be initiated at surfaces coated by a surfactant.

\footnotetext{
‘Corresponding Author andrew.goodwin@ colorado.edu. Present Addresses

†Adem Yildirim: CEDAR, Knight Cancer Institute, Oregon Health and Science University, Portland, OR 97239 USA

¥Angela Cai: Department of Chemistry, University of California, Berkeley, CA 94720 USA

${ }^{\wedge}$ Dennis Shi: Department of Chemical Engineering, Massachusetts Institute of Technology, Cambridge, MA 02142 USA

${ }^{+}$Rajarshi Chattaraj: Department of Chemical and Biomolecular Engineering, University of Pennsylvania, Philadelphia, PA 19104 USA

Author Contributions

The manuscript was written through contributions of all authors. All authors have given approval to the final version of the manuscript. ASSOCIATED CONTENT

Supporting Information. A PDF containing figures depicting the following: TEM images of hMSNs, HIFU setup and calibration curves, ultrasonographic images of hMSNs, DLS data of hMSNs with different lipid coverages, additional laurdan emission spectra, additional ultrasound contrast studies at an alternate particle concentration,. This material is available free of charge via the Internet at http://pubs.acs.org.
} 


\section{Graphical Abstract}

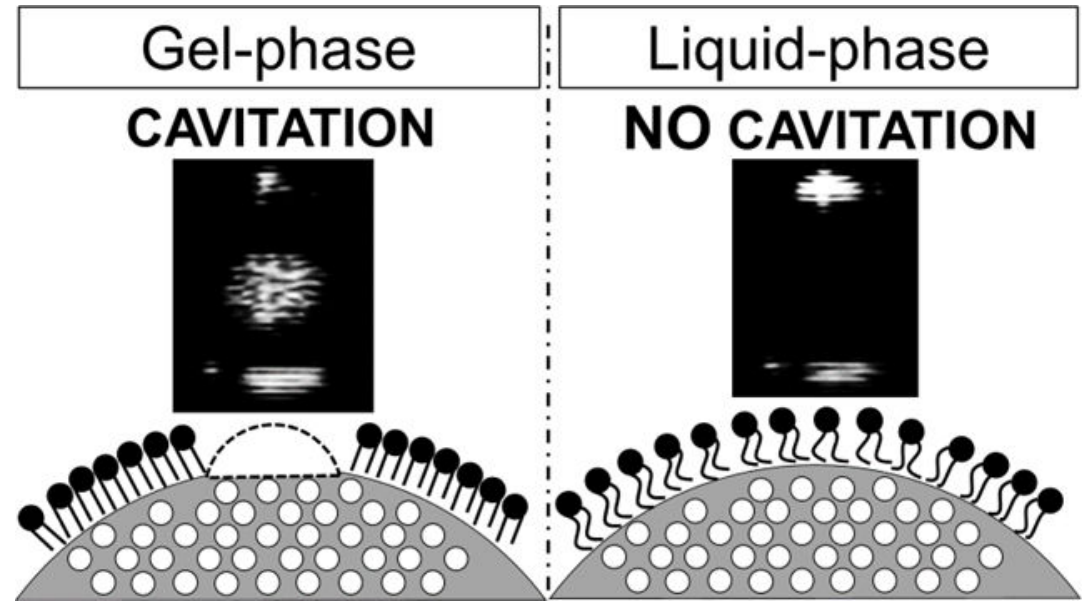

\section{Keywords}

Phospholipid; lateral phase separation; ultrasound; stimulus-responsive; nanoparticles

\section{Introduction.}

High intensity focused ultrasound (HIFU) has been utilized for many targeted therapy applications by leveraging thermal or mechanical effects on tissue. ${ }^{1-6}$ In thermal HIFU ablation, long duration HIFU pulses cause a significant increase in the focal zone temperature through ultrasound wave absorption, resulting in tissue destruction, irreversible necrosis of the focal tissue, and inflammation of the surrounding tissues. ${ }^{7-10}$ Mechanical effects on tissue may be obtained by inducing cavitation in the focal area, which in turn generates shock waves, water jets, and shear forces that can cause reversible tissue and cell damage. ${ }^{11-15}$ For safety reasons, the HIFU dose, or overall energy deposition as a result of HIFU exposure intensity and time, must be carefully regulated to avoid off-target tissue necrosis, poration, or scarring. Thus, in the clinic, long cooling periods must be introduced by between pulses to reduce off-target side effects. ${ }^{16,17}$

Recently, we reported contrast agents composed of phospholipid-stabilized hydrophobicallymodified mesoporous silica nanoparticles (hMSNs), which sensitize HIFU-induced cavitation by providing a site for heterogeneous gas nucleation rather than vaporizing an existing liquid. ${ }^{18,19}$ The gas nucleation generates transient bubbles that not only scatter and reflect impinging ultrasound waves but also collapse to produce additional broadband waves. Each of these modes can be detected during imaging. As with more common ultrasound contrast agents such as microbubbles or phase-shift nanodroplets, hMSNs can also help to limit energy deposition by reducing the acoustic intensity required for cavitation to occur. ${ }^{20-25}$ Unlike these agents, though, the use of a solid oxide rather than an encapsulated fluid allows formulation of hMSNs that are small enough to extravasate from tissue and are very stable in biological media. ${ }^{26-28}$ Low concentrations of hMSNs were shown to generate ultrasound contrast and biological effects through a nucleation-growth-cavitation mechanism 
by interacting with low duty cycle but high intensity ultrasound waves. ${ }^{18,19}$ These low duty cycle HIFU pulses are well designed for sensitizing histotripsy, which allows for less attenuation and less non-specific damage to surrounding tissue while still providing an enhanced localized therapeutic effect. ${ }^{29-32}$ Because the imaging and therapeutic effects of hMSN nucleated cavitation are mechanical rather than thermal in nature, even long exposures in controlled volumes do not lead to measurable bulk temperature rise at these conditions. ${ }^{30}$ However, like other contrast agents, the hMSNs cannot sense whether the tissue has reached a sufficient necrosis temperature.

This work describes mesoporous silica nanoparticle contrast agents that shut off their ultrasound contrast activity at necrosis temperature due to a phase change in their stabilizing phospholipid monolayer. As with many formulations of microbubbles and nanodroplets, phospholipids self-assemble into stable, biocompatible monolayers on hydrophobicallymodified silica nanoparticles. However, lipids are not simply passive bystanders, as they display rich phase behavior in solution at temperatures relevant to biological systems. Phosphocholines are known to undergo melting and freezing in bilayers and monolayers corresponding to their acyl tail length, with longer chained lipids have high melting temperatures. ${ }^{33,34}$ Consequently, longer lipids tend to adopt a gel phase, in which the lipids are condensed and tightly packed, while shorter or unsaturated lipids form a fluid phase, in which the tails may adopt more conformations and increase the effective packing area. ${ }^{35}$ When an interface is coated by a gel phase lipid, defects or holes can appear in the lipid monolayer due to imperfect packing of lipid islands on a curved surface, which in turn disappear when the lipid is heated above its melting temperature. ${ }^{36,37}$ In addition to temperature, certain surfactants, proteins, and other additives have been known to alter the packing and order of the lipid monolayer. ${ }^{38,39}$

In this work, we show not only that the phase of the lipid monolayer stabilizing the hMSNs (P@hMSNs) dictates the acoustic contrast, but that the lipid melting temperature can be used to impart temperature-sensitivity to the nanoparticles. This is a surprising result given that bubble heterogeneous nucleation is mainly dictated by the energy of the nucleating surface, which should not be significantly different whether the lipids are in a gel or liquid phase. ${ }^{40}$ We hypothesize that the difference in acoustic activity stems from the presence of lipid coating defects, holes, or inhomogeneities on the surface, and that these inhomogeneities resulting from gel phase lipids are crucial for initiating cavitation for mechanical HIFU ablation. We utilize this basic understanding to develop a proof-ofconcept mechanism for thermometric P@hMSNs that silence their acoustic activity in response to elevated temperatures and certain contaminants. Such materials may be useful in self-shut off therapeutic modalities, or as sensors for food packaging.

\section{Materials and Methods.}

\section{General materials.}

Tetraethylorthosilicate (TEOS) was purchased from Acros Organics. Dodecyltrichlorosilane was purchased from Gelest, Inc. Cetyltrimethylammonium chloride (CTAC, 25 wt.\% in water) was purchased from Sigma. Water was deionized with a Milli-Q Advantage A10 system (MilliporeSigma, Inc.) and was used without degassing. Chloroform and hexanes 
were purchased from Merck. Concentrated hydrochloric acid was obtained from Fischer Scientific. Bovine plasma was ordered from Lampire Biological Laboratories. 1,2dipalmitoyl-sn-glycero-3-phosphocholine (DPPC), 1,2-dibehenoyl-sn-glycero-3phosphocholine (DBPC), 1,2-dimyristoyl-sn-glycero-3-phosphocholine (DMPC), and 1,2dilauroyl-sn-glycero-3-phosphocholine (DLPC), were all purchased from Avanti Polar Lipids, Inc. 1,2-distearoyl-sn-glycero-3-phospho-ethanolamine-N-[methoxy(polyethylene glycol)- 2000] (DSPE-PEG2k) was also ordered from Avanti Polar Lipids, Inc.

\section{Synthesis of mesoporous silica nanoparticles (MSNs).}

The MSNs were prepared as described in previous publications via a modified Stober synthesis. ${ }^{18,19}$ Briefly, $5 \mathrm{~mL}$ of CTAC (Sigma) was dissolved in $15 \mathrm{~mL}$ deionized water and stirred at $600 \mathrm{rpm}$ at $75^{\circ} \mathrm{C}$ for $30 \mathrm{~min}$. Then, $0.8 \mathrm{~mL}$ of $10 \% \mathrm{v} / \mathrm{v}$ triethanolamine in deionized water was added and stirred at $80^{\circ} \mathrm{C}$ for another 45 min under a condenser. $1.5 \mathrm{~mL}$ of TEOS was then added dropwise. The reaction proceeded for $90 \mathrm{~min}$, after which the cloudy suspension was centrifuged at $7000 \mathrm{rcf}$ for $5 \mathrm{~min}$ and washed in ethanol twice. The CTAC was extracted from the nanoparticles by suspending the particles in acidic ethanol and stirring for $2 \mathrm{~h}$ at $65^{\circ} \mathrm{C}$, then centrifuging down again to remove the supernatant; this process was repeated once before centrifuge washing the particles twice with hexanes and then once with ethanol and drying at $65^{\circ} \mathrm{C}$ overnight in a water bath.

\section{Hydrophobic modification of MSNs.}

$50 \mathrm{mg}$ MSNs were baked in an oven at $120^{\circ} \mathrm{C}$ for $2 \mathrm{~h}$ in a $20 \mathrm{~mL}$ scintillation vial and suspended by sonicating vigorously for $5 \mathrm{~min}$ in $10 \mathrm{~mL}$ anhydrous DCM (Sigma) in a 50 $\mathrm{mL}$ round bottomed flask under argon. $300 \mu \mathrm{L}$ of dodecyltrichlorosililane was added and the reaction was stirred at $600 \mathrm{rpm}$ for $30 \mathrm{~h}$ before centrifuge washing twice with hexanes and once with ethanol at $7000 \mathrm{rcf}$ for $30 \mathrm{~min}$. The particles were dried overnight at $65^{\circ} \mathrm{C}$ in a water bath.

\section{Lipid coating of hMSNs.}

In order to coat the particles with lipids, $4.4 \mathrm{mg}$ of the hydrophobically modified particles were baked in a $20 \mathrm{~mL}$ glass scintillation vial at $120^{\circ} \mathrm{C}$ in an oven for $1 \mathrm{~h}$, allowed to cool, and then suspended in $1 \mathrm{~mL}$ chloroform. In the most typical formulation, $0.5 \mathrm{~mL}$ of a stock $4 \mathrm{mg} / \mathrm{mL}$ DPPC $(2.72 \mu \mathrm{mol})$ in chloroform was added, along with $0.3 \mathrm{~mL}$ of a $2 \mathrm{mg} / \mathrm{mL}$ DSPE-PEG2k $(0.21 \mu \mathrm{mol})$, so that the lipid and polymer-lipid comprised a 77:23\% mass ratio. The suspension was bath sonicated for $1 \mathrm{~min}$, then the chloroform was evaporated by drying the particles at $75^{\circ} \mathrm{C}$ in a water bath open to the atmosphere. The nanoparticle film was resuspended in $2 \mathrm{~mL}$ deionized water and bath sonicated for $3 \mathrm{~min}$, then transferred to a $4 \mathrm{~mL}$ glass vial and stirred at $75^{\circ} \mathrm{C}$ for $45 \mathrm{~min}$. The remaining solution was split into two 2$\mathrm{mL}$ centrifuge tubes, pelleted at 10,000 $\mathrm{rcf}$ for $5 \mathrm{~min}$, then resuspended to one $\mathrm{mL}$ each. The resulting dispersion was then split into four total tubes containing $0.5 \mathrm{~mL}$, and after addition of $0.5 \mathrm{~mL}$ of water in each, were briefly mixed then pelleted again with removal of the supernatant. The particles were stored as a $1 \mathrm{mg}$ pellet at room temperature for up to three days. When replacing DPPC as the main component of the coating, $2.72 \mu \mathrm{mol}$ of the alternate lipid was added, usually as a $4 \mathrm{mg} / \mathrm{mL}$ stock in chloroform, with the same amount 
of DSPE-PEG2k. The amount of chloroform was adjusted so that the final volume was always $1.8 \mathrm{~mL}$ before evaporation.

hMSN and P@hMSN structural characterizations.

An Anton Paar Litesizer 2000 was used to measure both the size distribution via dynamic light scattering (DLS) and zeta potential. For DLS, two $500 \mu \mathrm{L}$ aliquots of $1 \mathrm{mg} / \mathrm{mL}$ particle solutions in PBS, unless otherwise specified, from two separate batches were mixed to provide a representative scattering curve. The intensity weighted scattering curves were reported. Transmission electron microscopy (TEM) images of the nanoparticles were taken using a T12 Spirit (FEI Tecnai) microscope. TEM samples were prepped by dispersing the lipid-coated hydrophobically modified particles in $1 \%$ uranyl acetate aqueous solution and then drying them onto a carbon TEM grid. TEM images of the P@hMSNs can be found in the Supplemental Information as Figure S1 with a hard sphere diameter of about $\sim 110 \mathrm{~nm}$.

\section{HIFU setup and calibration.}

All components were placed in a plastic water bath of dimensions $2 \mathrm{ft}$ x $1 \mathrm{ft}$. A spherically focused, single-element, HIFU transducer (Sonic Concepts H101, $64.0 \mathrm{~mm}$ Active Diameter $\times 63.2 \mathrm{~mm}$ Radius of Curvature) with a coupling cone (Sonic Concepts C101) was used. The HIFU transducer was connected to a $30 \mathrm{MHz}$ Function/Arbitrary Waveform Generator (Agilent Technologies) through an AG Series Amplifier (T\&C Power Conversion, Inc.) with the amplifier operating at $100 \%$ output (Supplemental Figure S2). A 4V1 (Siemens Acuson Sequoia) transducer was aligned orthogonally to the direction of the HIFU apparatus, and this transducer was connected to a Sequoia ultrasound medical imager (Siemens Acuson 512) operating at, unless otherwise specified, in cadence contrast pulse sequencing mode, a frequency of $1.5 \mathrm{MHz}$, at mechanical index (MI) of 0.19 , and at a system gain of $18 \mathrm{~dB}$. The temperature in the bath was controlled by submerging a Vernatherm Bilbee Controls Ulanet Model 306 Immersion heater into the water tank in conjunction with a Digi-Sense Temperature Controller (Eutech Instruments Ptd Ltd). The water in the bath was kept uniform via a submerged Hydor Koralia Nano Aquarium Circulation Pump circulating water at a rate of 240 gallons per hour.

A needle hydrophone (HNC-0200, Onda Corp.) was used to calibrate the peak negative and positive pressures measured when the waveform generator was set to 12 cycles. The HIFU was operated at a center frequency of $1.1 \mathrm{MHz}$, and the peak negative pressures varied from 0 to $12 \mathrm{MPa}$, which was the maximum pressure achievable by our setup. Specific calibration data is given in the Supporting Information in Figure S3. Pressures above this maximum are reported at $12 \mathrm{MPa}$ in all graphs, with appropriate notation. The pulse repetition frequency was $10 \mathrm{~Hz}$ with a pulse duration of about $11 \mu \mathrm{s}$, resulting in a duty cycle of about $0.01 \%$.

\section{Measurement of P@hMSN onset peak negative pressure to HIFU.}

To interrogate the acoustic activity of the particles, a particle stock was made by suspending one of the $1 \mathrm{mg}$ pellets in $1 \mathrm{~mL}$ PBS, or in another aqueous medium as indicated in the main text, then diluted to the final concentration and solution composition. The solutions were gently mixed after dilution, then tested for onset acoustic pressure. In order to test for acoustic onset, the voltage on the waveform generated was slowly increased at a rate of 
approximately $100 \mathrm{mV}$ per 6 seconds in $10 \mathrm{mV}$ increments, or about $148 \mathrm{kPa}$ in the main linear regime (Supplemental Figure S3). The acoustic onset was measured when the particles gave detectable acoustic signal for at least $10 \mathrm{~s}$ as determined by imaging from the Siemens Sequoia ultrasound medical imager. Beyond acoustic onset pressures, the HIFU pulses caused the particles to cavitate consistently, giving a sonographic brightness comparable to that of commercial microbubbles (Supplemental Figure S4). Additionally, an audible snapping noise was observed that does not appear in the absence of acoustically active particles, signifying broadband acoustic emissions associated with inertial cavitation. 30,41,42 These observations, taken together, represent "cavitation" or "cavitation nucleation" for these studies: the growth of bubbles from the nanoparticle solution whose inherent compressibility and collapse allow for effective sonographic visualization, as reported previously. ${ }^{18,19,29}$ Typically, particles were tested within several minutes after dilution for each trial. Unless otherwise noted, displayed sonograms of the phantom were taken with an HIFU peak negative pressure of $10.8 \mathrm{MPa}$.

\section{Temperature variation studies.}

P@ hMSNs were suspended in PBS first to $1 \mathrm{mg} / \mathrm{mL}$ and then were diluted to either 100 $\mu \mathrm{g} / \mathrm{mL}$ or $20 \mu \mathrm{L}$ in either PBS or bovine plasma. The HIFU apparatus bath was heated to $20^{\circ} \mathrm{C}, 37^{\circ} \mathrm{C}$, or $55^{\circ} \mathrm{C}$ within a tolerance of $\pm 1^{\circ} \mathrm{C}$. The diluted particle solutions were then incubated in a temperature-controlled tank for $10 \mathrm{~min}$ to allow equilibration before onset of peak negative pressure was determined.

\section{Fluorescence characterization of lipid phase with laurdan.}

To fluorescently label the lipids, $10 \mathrm{mM}$ laurdan in ethanol was added to the chloroform containing lipid and hMSNs before drying to create a 0.01 laurdan to lipid molar ratio. The $\mathrm{P} @ \mathrm{hMSNs}$ were prepared as above, taking care to protect them from light. Fluorometer measurements were taken using a Fluoromax-4 Spectrofluorometer (Horiba Scientific, Irvine, CA) where the emission spectrum [400-600 nm] was taken at a specific excitation wavelength. For plots of multiple excitation wavelengths, this process was repeated at different excitation wavelengths in a randomized fashion. Generalized polarization was calculated as the difference in fluorescence emission intensity at 440 and $490 \mathrm{~nm}$, divided by the sum of these emission intensities.

\section{Results \& Discussion}

The overall hypothesis explaining the acoustic activity of P@hMSNs derives from the stabilization of incipient bubble nuclei on the surface of the nanoparticle, followed by growth to larger transient bubbles. ${ }^{18,19}$ As the nanoparticle surface properties become more favorable for bubble nucleation, typically by reducing the interfacial energy at the particlewater interface, the acoustic energy required to form detectable bubbles should decrease in accordance with the Kelvin effect. ${ }^{43,44}$ Thus, contrast activity can be correlated to the peak negative pressure (PNP) at which acoustic activity is detected by an ultrasound imager, or onset PNP. Here, a smaller onset PNP means that the P@hMSN has more acoustic contrast, while a larger onset PNP indicates that the P@hMSN may have little or no acoustic contrast. In these experiments, P@hMSNs were used that were stabilized by a saturated, two-tailed 
phosphocholine doped with $0.21 \mu \mathrm{mol}$ of DSPE-PEG, or $23 \%$ of total mass, to provide additional steric stabilization.

To understand the effects of lipid tail length and associated transition on acoustic contrast, the length of the lipid tail was varied from C12 (DLPC) to C22 (DBPC) while the headgroup was kept as a zwitterionic phosphocholine. For C14, C16, and C22 lipids, with corresponding melting temperatures of 24,41 , and $75^{\circ} \mathrm{C}$, all P@hMSN formulations had a measurable onset PNP (Figure 1). However, when the tail length was shortened to $\mathrm{C} 12$ (DLPC, $\mathrm{T}_{\mathrm{m}} \sim-2^{\circ} \mathrm{C}$ ), acoustic activity of the P@hMSNs could no longer be measured by our system, meaning that the onset PNP had increased beyond the transducer limit of $12 \mathrm{MPa}$. Of these lipids, only DLPC has a melting temperature below room temperature $\left(20^{\circ} \mathrm{C}\right)$, meaning that only DLPC would likely be present in a liquid expanded phase on the hMSN surface. To rule out the possibility of changes in aggregation, the particle size distributions for each lipid type were measured via DLS, and no significant differences were noted (Supplemental Figure S5). Expanding upon the hypothesis that lipid phase dictated acoustic activity, we next studied the effect of temperature on the onset PNP by varying the temperature of the water bath. While we see non-significant differences for DBPC and DPPC in terms of their onset PNP, DMPC exhibited an increasing onset PNP with increasing temperature, showing total acoustic deactivation at $55^{\circ} \mathrm{C}$. As expected, DLPC remained inactive throughout. While the quenching of acoustic contrast at $55^{\circ} \mathrm{C}$ does not align exactly with the melting temperature of DMPC $\left(24^{\circ} \mathrm{C}\right)$, these melting temperatures are measured in bulk and do not take into account any effects of the monolayer arrangement or the curvature of the nanoparticle surface. ${ }^{45,46}$

Next, fluorescence spectroscopy was conducted with laurdan dye to verify that phase change of the lipid was indeed occurring concomitantly with the acoustic contrast of the P@hMSNs. Laurdan is a hydrophobic membrane stain that undergoes a large red shift, about $50 \mathrm{~nm}$, in both emission and excitation wavelengths during the transition from gel to fluid lipid phase. 48,49 This shift occurs due to the increased penetration of the water molecules into the lipid layer, and whose dipole-diploe interaction causes the red-shift in the laurdan dye. ${ }^{50,51}$ Thus, as membrane fluidity increases, laurdan emission shifts further to the red. For DPPC coated hMSNs with $1 \mathrm{~mol} \%$ laurdan, the peak of the emission spectrum shifted from $446 \mathrm{~nm}$ at $20^{\circ} \mathrm{C}$ to $450 \mathrm{~nm}$ at $37^{\circ} \mathrm{C}$ to 468 at $55^{\circ} \mathrm{C}$ (Figure 2). The background scattering contribution of particles was negligible under these conditions (Supplemental Figure S6). To show the extent of shifting, generalized polarization (GP) of laurdan was calculated as the ratio of the difference of emission intensities at $440 \mathrm{~nm}$ and $490 \mathrm{~nm}$ for a given excitation wavelength over the sum of the same emission intensities (Figure 2), where a lower smaller GP value corresponds to laurdan embedded in a more fluid membrane. ${ }^{52} \mathrm{GP}$ was calculated at a range of excitation wavelengths to account for any variations in GP; however, in this system GP was essentially independent of excitation wavelength (Figure 2). As expected, a GP >0 was found at temperatures below DPPC's $\mathrm{T}_{\mathrm{m}}$ at $\sim 41^{\circ} \mathrm{C}$, while $\mathrm{GP} \sim 0$ was found at temperatures above DMPC's $\mathrm{T}_{\mathrm{m}}$ at $\sim 24^{\circ} \mathrm{C}$, corresponding to the expected phase (Supplemental Figure S7). Thus, it was observed that increasing temperature led to reduced acoustic activity of P@hMSNs, where the greatest change in acoustic activity occurred near the gel-fluid phase transition temperature of each phospholipid. ${ }^{33-35}$ 
Having established a correlation between lipid phase and temperature, we next studied the effect of adding chemical fluidizers to the lipid monolayer. Certain amphiphiles such as Triton $\mathrm{X}$ induce membrane fluidity via lipid monolayer destabilization, and thus should inactivate the P@hMSNs. ${ }^{38}$ To test this hypothesis, $1 \mathrm{mg} / \mathrm{mL}$ of P@hMSNs suspended in PBS was incubated for $2 \mathrm{~h}$ at RT with $3 \mathrm{mM}$ of Triton $\mathrm{X}$, the particles were centrifuge washed once, and finally their acoustic activity was tested. Triton $\mathrm{X}$ eliminated acoustic activity in the nanoparticle beyond the testing limits of our system (Figure 3A). However, this quenching could be reversed: centrifuge washing the nanoparticles three times in PBS caused acoustic activity to return for the Triton X sample only to an onset PNP of $6 \mathrm{MPa}$, which was similar to nanoparticles without additive but treated to the same wash conditions (Figure 3A). While nanoparticle dispersibility as measured by DLS was initially stable during Triton $\mathrm{X}$ challenge, subsequent washing caused significant aggregation (Figure 3B) as compared to the PBS control (Supplemental Figure S8), presumably because of monolayer destabilization by Triton X. Thus, the quenching of acoustic activity appeared to be reversible, even if the nanoparticles themselves did not retain their overall dispersibility.

Similarly, it is known that blood plasma contains many proteins and molecules with high affinity for nanoparticles, particularly nanoparticles with hydrophobic surfaces. ${ }^{53}$ To test how these proteins might potentially impact the lipid phase on P@hMSNs and their resulting acoustic contrast, the acoustic activity of P@hMSNs was evaluated in the presence of $50 \%$ citrate-stabilized bovine plasma after a one hour incubation period. While only a small increase in the onset PNP was observed for DPPC coated particles, a larger increase of $3 \mathrm{MPa}$ was observed in the onset PNP of DMPC (Figure 4A). A corresponding decrease in acoustic imaging signal was recorded when imaging at HIFU PNP of $12 \mathrm{MPa}$ (Figure 4B). Video brightness analysis was conducted for some of these samples and the results plotted in the Supporting Information as Figure S9. To compare acoustic tests with lipid phase behavior, we compared laurdan fluorescence in DPPC-coated P@hMSNs in the presence of either Triton X or bovine plasma. Both the laurdan emission spectra and the GP values indicate a minor effect on acoustic contrast due to incubation with plasma (Figure 4C) but a larger effect when exposed to Triton X, again matching the acoustic contrast data (Figure 4C). Taking these results together, the presence of plasma appears to affect the acoustic contrast by partially fluidizing the lipid monolayer, but not to an extent at which acoustic contrast is quenched completely. ${ }^{47}$

In mixed lipid systems, the presence of lipids with different tail lengths can lead to complex phase behavior with multiple coexisting phases ${ }^{54-57}$ To test the combination of both gelforming and liquid-forming lipids on acoustic contrast, DLPC (C12) was mixed with DPPC (C16) at $1 \%, 10 \%$, and $25 \%$ mol\%. While $1 \%$ mol fraction DLPC was not sufficient to quench acoustic activity, at 10\% DLPC the onset PNP rose significantly, and at 25\%, the acoustic response was fully quenched (Figure 5A). Once again, the dispersibility of these particle suspensions were not significantly different from each other (Figure S10). In comparing nanoparticles stabilized by pure DMPC, DPPC, and DBPC, addition of $50 \mathrm{~mol} \%$ DLPC completely quenched all imaging signal at a PNP of $12 \mathrm{MPa}$, indicating a total absence of cavitation (Figure 5B). Because 50\% DLPC appears to quench all other lipid compositions, the reduction in acoustic contrast appears to occur even if gel islands form on the nanoparticle surface. 
Finally, these results indicate the ability to utilize the P@hMSNs for self-regulated thermometry during application of HIFU. Figure 6 shows the onset activity of P@hMSNs with different lipid coatings at $20^{\circ} \mathrm{C}, 37^{\circ} \mathrm{C}$, and $55^{\circ} \mathrm{C}$ with PNPs tested in $90 \%$ citratestabilized bovine plasma. At a constant HIFU intensity of 10.4 MPa, DPPC-stabilized $\mathrm{P} @ \mathrm{hMSNs}$ could differentiate between $37^{\circ} \mathrm{C}$ and $55^{\circ} \mathrm{C}$. These and other trends were replicated at particle concentrations of $20 \mu \mathrm{g} / \mathrm{mL}$ (Supplemental Figure S11) with a notable increase in onset acoustic pressure correlating to the reduced number of cavitation nucleation sites at the smaller particle concentration. To our knowledge, this result is the first example of a nanoscale temperature-sensing HIFU-responsive particle that does not rely upon aggregation to modulate acoustic activity. Interestingly, DMPC became more fluid at higher plasma concentration, indicating that possibly a weak corona formation could drive loss of acoustic contrast. Thus, in designing future thermometric self-limiting HIFU contrast agents, adjustments must be made from the literature melting temperature in order to finetune the turn-off temperature.

\section{Conclusion.}

In summary, we have correlated the lateral phase of the lipid monolayer coating the particles, as determined by laurdan fluorescence, to the ability of a hydrophobic nanoparticle solution to initiate cavitation in response to HIFU. While the Kelvin effect should dictate that only a hydrophobic surface is necessary to promote bubble nucleus formation, the behavior of the phospholipid monolayer must also be considered. It is known, for example, that surface-stabilized nanobubbles form on hydrophobic surfaces but also require the presence of topological defects to pin their contact line. ${ }^{58,59}$ Our hypothesis is that a gel phase lipid monolayer forms defects when forced to conform to the high curvature of the nanoparticles (radius $\sim 150 \mathrm{~nm}$ ). These cone-like defects are ideal geometries for stabilizing gas pockets along the surface of the particle and the lipid tail (Figure 7), which in turn are suitable nuclei for heterogeneous initiation of cavitation. ${ }^{60}$ This interpretation bolstered by atomic force microscopy studies of supported lipid layers, which show holes and defects in gel phase lipid layers as compared to the same layers when fluidized. ${ }^{61}$ In the presence of contaminants, the defect sites can be filled by the fluidizing groups, so that even if the lipid does not lose its gel phase the signal is still quenched. Taken together, our results placed in the context of existing literature show a plausible mechanism for how P@hMSNs promote acoustic contrast as a function of lipid phase. Based on this new understanding of heterogeneous cavitation on nanoparticles for low duty cycle HIFU, we have designed temperature responsive contrast agents that can operate both in saline and plasma. This work facilitates the use of these particles as "switch off' agents for limiting undesired damage in HIFU therapeutic applications.

\section{Supplementary Material}

Refer to Web version on PubMed Central for supplementary material.

\section{ACKNOWLEDGMENT}

The authors thank the National Institute of Biomedical Imaging and Bioengineering (NIBIB) for support of this research through grants DP2EB020401, R03EB021432, and EB026006. Additionally, the authors thank Prof. 
Natalie Ahn for use of laurdan dye, and Prof. Daniel Schwartz for use of his fluorometer and James Weltz for assistance in its operation. The authors also thank Prof. Theodore Randolph for use of his Anton Paar Litesizer. Finally, the authors acknowledge Prof. Dmitri Simberg of University of Colorado Denver for helpful suggestions.

Funding Sources

NIH DP2EB020401, NIH R03EB021432, NIH R21EB026006.

\author{
ABBREVIATIONS \\ CTAC cetyltrimethylammonium chloride \\ DBPC 1,2-dibehenoyl-sn-glycero-3-phosphocholine \\ DCM dichloromethane \\ DLPC 1,2-dilauroyl-sn-glycero-3-phosphocholine \\ DMPC 1,2-dimyristoyl-sn-glycero-3-phosphocholine \\ DPPC 1,2-dipalmitoyl-sn-glycero-3-phosphocholine \\ DSPE-PEG2k 1,2-distearoyl-sn-glycero-3-phospho-ethanolamine-N- \\ [methoxy(polyethylene glycol)-2000] \\ GP generalized polarization \\ HIFU high intensity focused ultrasound \\ hMSN hydrophobic mesoporous silica nanoparticles \\ MI mechanical index \\ P@hMSN phospholipid coated hydrophobic mesoporous silica \\ nanoparticles \\ PNP peak negative/rarefactional pressure \\ TEOS tetraethylorthosilicate
}

\title{
REFERENCES
}

(1). Diederich CJ Thermal Ablation and High-Temperature Thermal Therapy: Overview of Technology and Clinical Implementation. Int. J. Hyperthermia 2005, 21 (8), 745-753. [PubMed: 16338857]

(2). Chu KF; Dupuy DE Thermal Ablation of Tumours: Biological Mechanisms and Advances in Therapy. Nat. Rev. Cancer 2014, 14 (3), 199-208. [PubMed: 24561446]

(3). Jang HJ; Lee J-Y; Lee D-H; Kim W-H; Hwang JH Current and Future Clinical Applications of High-Intensity Focused Ultrasound (HIFU) for Pancreatic Cancer. Gut Liver 2010, 4 (Suppl 1), S57-S61. [PubMed: 21103296]

(4). Roberts WW; Hall TL; Ives K; Wolf JS; Fowlkes JB; Cain CA Pulsed Cavitational Ultrasound: A Noninvasive Technology for Controlled Tissue Ablation (Histotripsy) in the Rabbit Kidney. J. Urol. 2006, 175 (2), 734-738. [PubMed: 16407041]

(5). Köhler MO; Mougenot C; Quesson B; Enholm J; Bail BL; Laurent C; Moonen CTW; Ehnholm GJ Volumetric HIFU Ablation under 3D Guidance of Rapid MRI Thermometry. Med. Phys. 2009, 36 (8), 3521-3535. [PubMed: 19746786] 
(6). Siegal RJ; Vaezy S; Martin R; Crum L Therapeutic Ultrasound, Part II*. High Intensity Focused Ultrasound: A Method of Hemostasis. Echocardiography 2001, 18 (4), 309-315. [PubMed: 11415503]

(7). Wu F; Wang Z-B; Cao Y-D; Chen W-Z; Bai J; Zou J-Z; Zhu H A Randomised Clinical Trial of High-Intensity Focused Ultrasound Ablation for the Treatment of Patients with Localised Breast Cancer. Br. J. Cancer 2003, 89, 2227-2233. [PubMed: 14676799]

(8). Wu F Extracorporeal High Intensity Focused Ultrasound in the Treatment of Patients with Solid Malignancy. Minim. Invasive Ther. Allied Technol. 2006, 15 (1), 26-35. [PubMed: 16703687]

(9). Nikfarjam M; Muralidharan V; Christophi C Mechanisms of Focal Heat Destruction of Liver Tumors. J. Surg. Res. 2005, 127 (2), 208-223. [PubMed: 16083756]

(10). Elhelf IAS; Albahar H; Shah U; Oto A; Cressman E; Almekkawy M High Intensity Focused Ultrasound: The Fundamentals, Clinical Applications and Research Trends. Diagn. Interv. Imaging 2018, 99 (6), 349-359. [PubMed: 29778401]

(11). Hoogenboom M; van Amerongen MJ; Eikelenboom DC; Wassink M; den Brok MH; Hulsbergenvan de Kaa C; Dumont E; Adema GJ; Heerschap A; Fütterer JJ Development of a High-Field MR-Guided HIFU Setup for Thermal and Mechanical Ablation Methods in Small Animals. J. Ther. Ultrasound 2015, 3 (1), 14. [PubMed: 26269744]

(12). Hu Z; Yang XY; Liu Y; Sankin GN; Pua EC; Morse MA; Lyerly HK; Clay TM; Zhong P Investigation of HIFU-Induced Anti-Tumor Immunity in a Murine Tumor Model. J. Transl. Med. 2007, 5 (1), 34. [PubMed: 17625013]

(13). Coussios CC; Farny CH; Haar GT; Roy RA Role of Acoustic Cavitation in the Delivery and Monitoring of Cancer Treatment by High-Intensity Focused Ultrasound (HIFU). Int. J. Hyperthermia 2007, 23 (2), 105-120. [PubMed: 17578336]

(14). O’Neill BE; Vo H; Angstadt M; Li KPC; Quinn T; Frenkel V Pulsed High Intensity Focused Ultrasound Mediated Nanoparticle Delivery: Mechanisms and Efficacy in Murine Muscle. Ultrasound Med. Biol. 2009, 35 (3), 416-424. [PubMed: 19081668]

(15). Mesiwala AH; Farrell L; Wenzel HJ; Silbergeld DL; Crum LA; Winn HR; Mourad PD HighIntensity Focused Ultrasound Selectively Disrupts the Blood-Brain Barrier in Vivo. Ultrasound Med. Biol. 2002, 28 (3), 389-400. [PubMed: 11978420]

(16). Hancock HA; Smith LH; Cuesta J; Durrani AK; Angstadt M; Palmeri ML; Kimmel E; Frenkel V Investigations into Pulsed High-Intensity Focused Ultrasound-Enhanced Delivery: Preliminary Evidence for a Novel Mechanism. Ultrasound Med. Biol. 2009, 35 (10), 1722-1736. [PubMed: 19616368]

(17). Poff JA; Allen CT; Traughber B; Colunga A; Xie J; Chen Z; Wood BJ; Van Waes C; Li KCP; Frenkel V Pulsed High-Intensity Focused Ultrasound Enhances Apoptosis and Growth Inhibition of Squamous Cell Carcinoma Xenografts with Proteasome Inhibitor Bortezomib. Radiology 2008, 248 (2), 485-491. [PubMed: 18574138]

(18). Yildirim A; Chattaraj R; Blum NT; Goldscheitter GM; Goodwin AP Stable Encapsulation of Air in Mesoporous Silica Nanoparticles: Fluorocarbon-Free Nanoscale Ultrasound Contrast Agents. Adv. Healthc. Mater. 2016, 5 (11), 1290-1298. [PubMed: 26990167]

(19). Yildirim A; Chattaraj R; Blum NT; Goodwin AP Understanding Acoustic Cavitation Initiation by Porous Nanoparticles: Toward Nanoscale Agents for Ultrasound Imaging and Therapy. Chem. Mater. 2016, 28 (16), 5962-5972. [PubMed: 28484307]

(20). Zhou Y; Wang Z; Chen Y; Shen H; Luo Z; Li A; Wang Q; Ran H; Li P; Song W; et al. Microbubbles from Gas-Generating Perfluorohexane Nanoemulsions for Targeted TemperatureSensitive Ultrasonography and Synergistic HIFU Ablation of Tumors. Adv. Mater. 2013, 25 (30), 4123-4130. [PubMed: 23788403]

(21). Zhang P; Porter T An in Vitro Study of a Phase-Shift Nanoemulsion: A Potential Nucleation Agent for Bubble-Enhanced HIFU Tumor Ablation. Ultrasound Med. Biol. 2010, 36 (11), 18561866. [PubMed: 20888685]

(22). Lin C-Y; Pitt WG Acoustic Droplet Vaporization in Biology and Medicine. Biomed. Res. Intl. 2013, 40436.

(23). Farny* CH; Holt RG; Roy RA The Correlation Between Bubble-Enhanced HIFU Heating and Cavitation Power. IEEE Trans. Biomed. Eng. 2010, 57 (1), 175-184. [PubMed: 19651548] 
(24). Hoff L Acoustic Characterization of Contrast Agents for Medical Ultrasound Imaging, 1st.; Kluwer Academic Publishers: Boston, USA, 2011.

(25). Shpak O; Verweij M; Vos HJ; Jong N de; Lohse, D.; Versluis, M. Acoustic Droplet Vaporization Is Initiated by Superharmonic Focusing. Proc. Natl. Acad. Sci. 2014, 111 (5), 1697-1702. [PubMed: 24449879]

(26). Landfester K On the Stability of Liquid Nanodroplets in Polymerizable Miniemulsions. J. Dispers. Sci. Technol. 2002, 23 (1-3), 167-173.

(27). Rapoport N Phase-Shift, Stimuli-Responsive Perfluorocarbon Nanodroplets for Drug Delivery to Cancer. Wiley Interdiscip. Rev. Nanomed. Nanobiotechnol. 2012, 4 (5), 492-510.

(28). Jain RK; Stylianopoulos T Delivering Nanomedicine to Solid Tumors. Nat. Rev. Clin. Oncol. 2010, 7 (11), 653-664. [PubMed: 20838415]

(29). Yildirim A; Chattaraj R; Blum NT; Shi D; Kumar K; Goodwin AP Phospholipid Capped Mesoporous Nanoparticles for Targeted High Intensity Focused Ultrasound Ablation. Adv. Healthc. Mater. 2017, 6 (18), 1700514.

(30). Yildirim A; Shi D; Roy S; Blum NT; Chattaraj R; Cha JN; Goodwin AP Nanoparticle-Mediated Acoustic Cavitation Enables High Intensity Focused Ultrasound Ablation Without Tissue Heating. ACS Appl. Mater. Interfaces 2018.

(31). Chen Y; Gao Y; Chen H; Zeng D; Li Y; Zheng Y; Li F; Ji X; Wang X; Chen F; et al. Engineering Inorganic Nanoemulsions/Nanoliposomes by Fluoride-Silica Chemistry for Efficient Delivery/Co-Delivery of Hydrophobic Agents. Adv. Funct. Mater. 2012, 22 (8), 1586-1597.

(32). Wang X; Chen H; Chen Y; Ma M; Zhang K; Li F; Zheng Y; Zeng D; Wang Q; Shi J Perfluorohexane-Encapsulated Mesoporous Silica Nanocapsules as Enhancement Agents for Highly Efficient High Intensity Focused Ultrasound (HIFU). Adv. Mater. 2012, 24 (6), 785-791. [PubMed: 22223403]

(33). Sun WJ; Tristram-Nagle S; Suter RM; Nagle JF Structure of Gel Phase Saturated Lecithin Bilayers: Temperature and Chain Length Dependence. Biophys. J. 1996, 71 (2), 885-891. [PubMed: 8842227]

(34). Jacobson K; Papahadjopoulos D Phase Transitions and Phase Separations in Phospholipid Membranes Induced by Changes in Temperature, PH, and Concentration of Bivalent Cations. Biochemistry 1975, 14 (1), 152-161. [PubMed: 234017]

(35). Marsh D General Features of Phospholipid Phase Transitions. Chem. Phys. Lipids 1991, 57 (2), 109-120.

(36). Charrier A; Thibaudau F Main Phase Transitions in Supported Lipid Single-Bilayer. Biophys. J. 2005, 89 (2), 1094-1101. [PubMed: 15879467]

(37). Keller D; Larsen NB; Möller IM; Mouritsen OG Decoupled Phase Transitions and GrainBoundary Melting in Supported Phospholipid Bilayers. Phys. Rev. Lett. 2005, 94 (2), 02570.

(38). Goñi FM; Urbaneja M-A; Arrondo JLR; Alonso A; Durrani AA; Chapman D The Interaction of Phosphatidylcholine Bilayers with Triton X-100. Eur. J. Biochem. 1986, 160 (3), 659-665. [PubMed: 3780729]

(39). Brockman H Lipid Monolayers: Why Use Half a Membrane to Characterize Protein-Membrane Interactions? Curr. Opin. Struct. Biol. 1999, 9 (4), 438-443. [PubMed: 10449364]

(40). Pinazo A; Wen X; Liao Y-C; Prosser AJ; Franses EI Comparison of DLPC and DPPC in Controlling the Dynamic Adsorption and Surface Tension of Their Aqueous Dispersions. Langmuir 2002, 18 (23), 8888-8896.

(41). Bull V; Civale J; Rivens I; ter Haar GR Cavitation Detection Using a Fibre-Optic Hydrophone: A Pilot Study. AIP Conf. Proc. 2011, 2359 (1), 85-90.

(42). Brayman AA; Chen W; Matula TJ; Crum LA; Miller MW The Pulse Duration Dependence of Inertial Cavitation Dose and Hemolysis. In 2002 IEEE Ultrasonics Symposium, 2002. Proceedings; 2002; Vol. 2, pp 1387-1390 vol.2.

(43). Lubetkin SD Why Is It Much Easier To Nucleate Gas Bubbles than Theory Predicts? Langmuir 2003, 19 (7), 2575-2587.

(44). Oxtoby DW Homogeneous Nucleation: Theory and Experiment. J. Phys. Condens. Matter 1992, $4(38), 7627$. 
(45). Chapman D; Urbina J; Keough KM Biomembrane Phase Transitions: Studies of Lipid-Water Systems Using Differential Scanning Calorimetry. J. Biol. Chem. 1974, 249 (8), 2512-2521. [PubMed: 4132554]

(46). Marrink SJ; Risselada J; Mark AE Simulation of Gel Phase Formation and Melting in Lipid Bilayers Using a Coarse Grained Model. Chem. Phys. Lipids 2005, 135 (2), 223-244. [PubMed: 15921980]

(47). Silvius J Thermotropic Phase Transitions of Pure Lipids in Model Membranes and Their Modifications by Membrane Proteins; John Wiley \& Sons, Inc: New York, 1982.

(48). Parasassi T; De Stasio G; Ravagnan G; Rusch RM; Gratton E Quantitation of Lipid Phases in Phospholipid Vesicles by the Generalized Polarization of Laurdan Fluorescence. Biophys. J. 1991, 60 (1), 179-189. [PubMed: 1883937]

(49). Parasassi T; Gratton E; Yu WM; Wilson P; Levi M Two-Photon Fluorescence Microscopy of Laurdan Generalized Polarization Domains in Model and Natural Membranes. Biophys. J. 1997, 72 (6), 2413-2429. [PubMed: 9168019]

(50). Zhang Y-L; Frangos JA; Chachisvilis M Laurdan Fluorescence Senses Mechanical Strain in the Lipid Bilayer Membrane. Biochem. Biophys. Res. Commun. 2006, 347 (3), 838-841. [PubMed: 16857174]

(51). Parasassi T; Di Stefano M; Loiero M; Ravagnan G; Gratton E Cholesterol Modifies Water Concentration and Dynamics in Phospholipid Bilayers: A Fluorescence Study Using Laurdan Probe. Biophys. J. 1994, 66 (3, Part 1), 763-768. [PubMed: 8011908]

(52). Parasassi T; De Stasio G; d'Ubaldo A; Gratton E Phase Fluctuation in Phospholipid Membranes Revealed by Laurdan Fluorescence. Biophys. J. 1990, 57 (6), 1179-1186. [PubMed: 2393703]

(53). Aggarwal P; Hall JB; McLeland CB; Dobrovolskaia MA; McNeil SE Nanoparticle Interaction with Plasma Proteins as It Relates to Particle Biodistribution, Biocompatibility and Therapeutic Efficacy. Adv. Drug Deliv. Rev. 2009, 61 (6), 428-437. [PubMed: 19376175]

(54). Veatch SL; Polozov IV; Gawrisch K; Keller SL Liquid Domains in Vesicles Investigated by NMR and Fluorescence Microscopy. Biophys. J. 2004, 86 (5), 2910-2922. [PubMed: 15111407]

(55). Chattaraj R; M. Goldscheitter G; Yildirim A; P. Goodwin A Phase Behavior of Mixed Lipid Monolayers on Perfluorocarbon Nanoemulsions and Its Effect on Acoustic Contrast. RSC Adv. 2016, 6 (112), 111318-111325. [PubMed: 28603605]

(56). Parasassi T; De Stasio G; d'Ubaldo A; Gratton E Phase Fluctuation in Phospholipid Membranes Revealed by Laurdan Fluorescence. Biophys. J. 1990, 57 (6), 1179-1186. [PubMed: 2393703]

(57). Parasassi T; Di Stefano M; Loiero M; Ravagnan G; Gratton E Cholesterol Modifies Water Concentration and Dynamics in Phospholipid Bilayers: A Fluorescence Study Using Laurdan Probe. Biophys. J. 1994, 66 (3, Part 1), 763-768. [PubMed: 8011908]

(58). Bull DS; Nelson N; Konetski D; Bowman CN; Schwartz DK; Goodwin AP Contact Line Pinning Is Not Required for Nanobubble Stability on Copolymer Brushes. J. Phys. Chem. Lett. 2018, 9 (15), 4239-4244. [PubMed: 30010342]

(59). Borkent BM; Dammer SM; Schönherr H; Vancso GJ; Lohse D Superstability of Surface Nanobubbles. Phys. Rev. Lett. 2007, 98 (20), 204502.

(60). Wilt PM Nucleation Rates and Bubble Stability in Water-Carbon Dioxide Solutions. J. Colloid Interface Sci. 1986, 112 (2), 530-538.

(61). Garcia-Manyes S; Sanz F Nanomechanics of Lipid Bilayers by Force Spectroscopy with AFM: A Perspective. Biochim. Biophys. Acta BBA - Biomembr. 2010, 1798 (4), 741-749. 

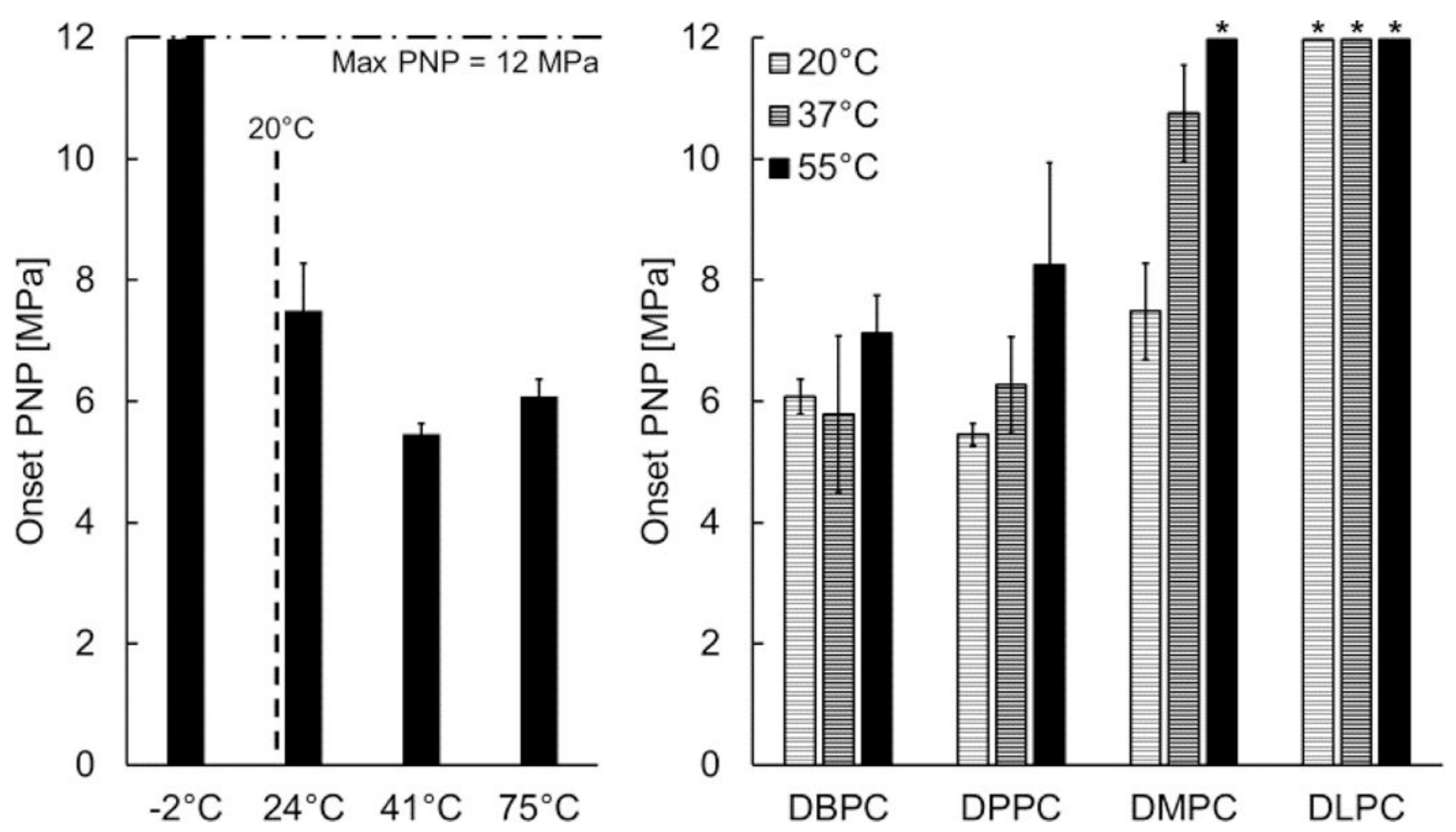

Figure 1.

(Left) onset, or required, peak negative pressure (PNP) to initiate cavitation nucleation from $100 \mathrm{ng} / \mathrm{mL}$ P@hMSNs of increasing melting temperature transitions: DLPC, $-2^{\circ} \mathrm{C}$; DMPC, $24^{\circ} \mathrm{C}$; DPPC, $41^{\circ} \mathrm{C}$; DBPC, $75^{\circ} \mathrm{C} .4^{47}$ The temperature at which experiments were carried out is denoted by the dashed line at $\sim 20^{\circ} \mathrm{C}$. The dash-dotted line at $12 \mathrm{MPa}$ denotes the maximum PNP achievable by our system. (Right) Onset peak negative pressure (PNP) for lipid formulations of (left to right) decreasing tail length: DBPC, C22; DPPC, C16; DMPC, C14; DLPC, C12, and increasing temperature. All error bars denote one standard deviation. Bars with stars above them denote that no acoustic activity was observed at the maximum PNP of $12 \mathrm{MPa}$. 


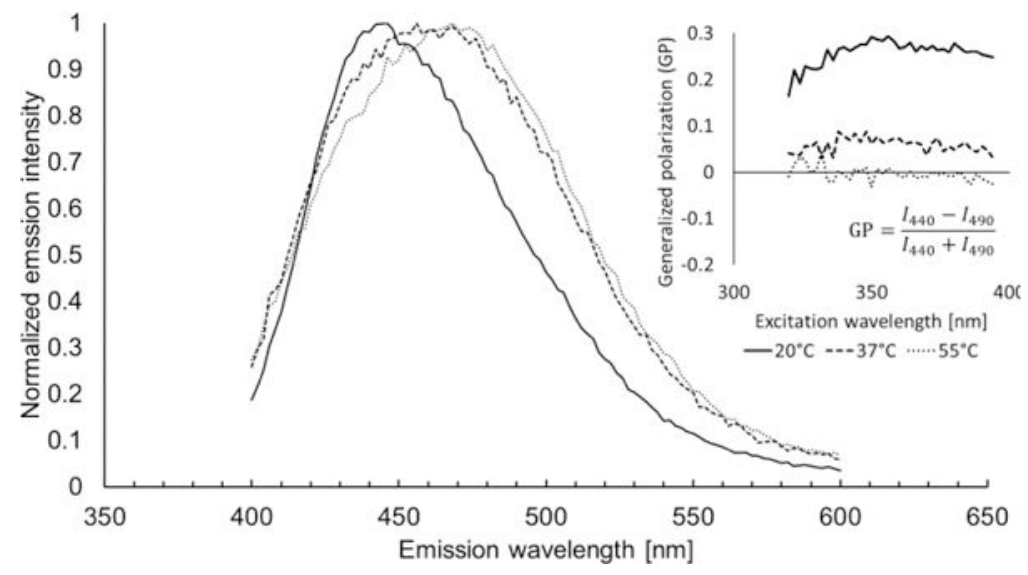

Figure 2.

Laurdan fluorescence emission spectra for DPPC P@hMSNs at an excitation of $370 \mathrm{~nm}$ for samples at $20^{\circ} \mathrm{C}, 37^{\circ} \mathrm{C}$, and $55^{\circ} \mathrm{C}$. Inset: Generalized polarization (GP) equation and values for laurdan calculated plotted for DPPC P@hMSNs at different excitation wavelengths. 

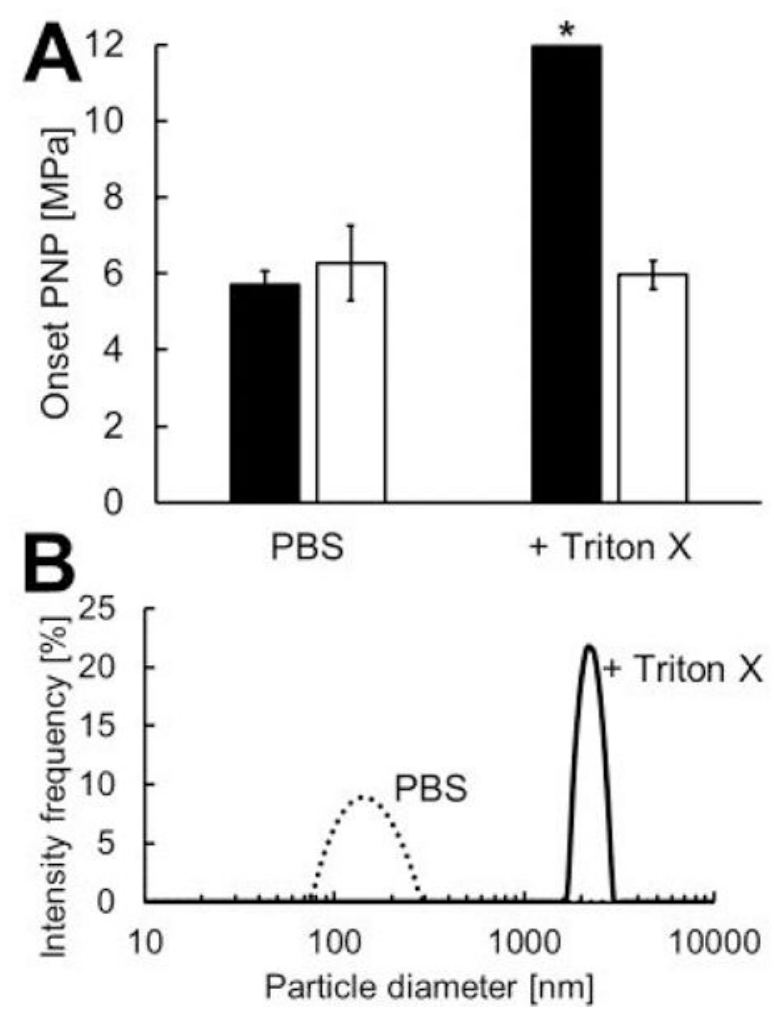

Figure 3.

(A) Onset PNP of P@hMSNs at $100 \mu \mathrm{g} / \mathrm{mL}$ that were incubated in either PBS or PBS with 3 $\mathrm{mM}$ Triton $\mathrm{X}$ and washed once (dark bar) or four times (light bar). (B) Dynamic light scattering (DLS) showing size distribution of nanoparticles washed four times total. Error bars denote one standard deviation; bars with stars above them denote that no acoustic activity was observed at the maximum PNP of $12 \mathrm{MPa}$. 

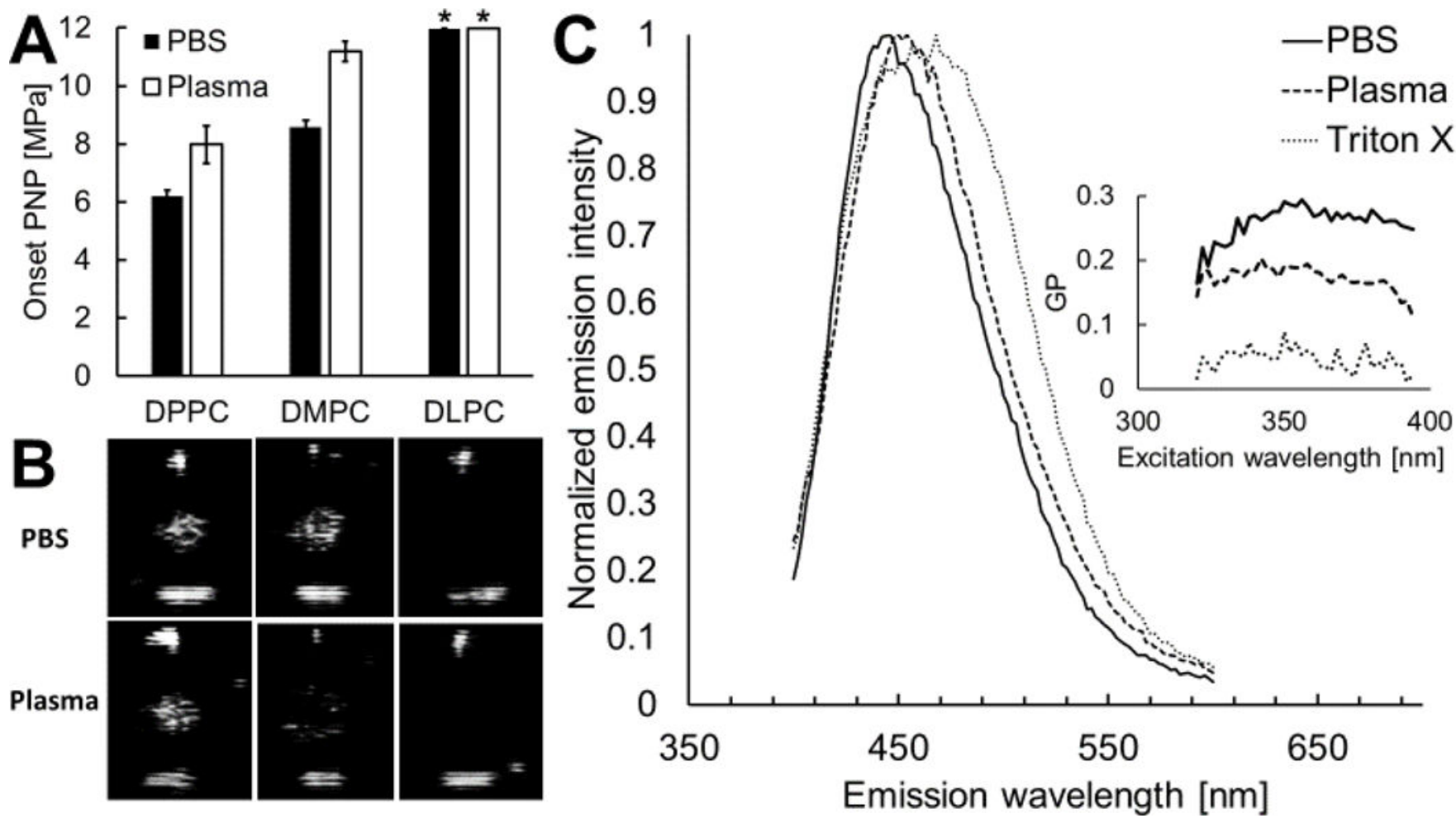

Figure 4.

(A) Shows the onset PNP for DPPC, DMPC, and DLPC coated hMSNs in PBS and bovine plasma at a concentration of $100 \mu \mathrm{g} / \mathrm{mL}$. (B) Medical ultrasound images taken at $10.8 \mathrm{MPa}$ PNP HIFU of the corresponding particles in a phantom. For reference, the cross-sectional diameter of the phantom (bound by bright lines at the top and bottom of the image) is 12 $\mathrm{mm}$. (C) Emission spectra of laurdan doped DPPC coated hMSNs in PBS, plasma, and 3 mM Triton X PBS solution and (inset) corresponding GP values plotted versus excitation wavelength. Error bars denote one standard deviation; bars with stars above them denote that no acoustic activity was observed at the maximum PNP of $12 \mathrm{MPa}$. 

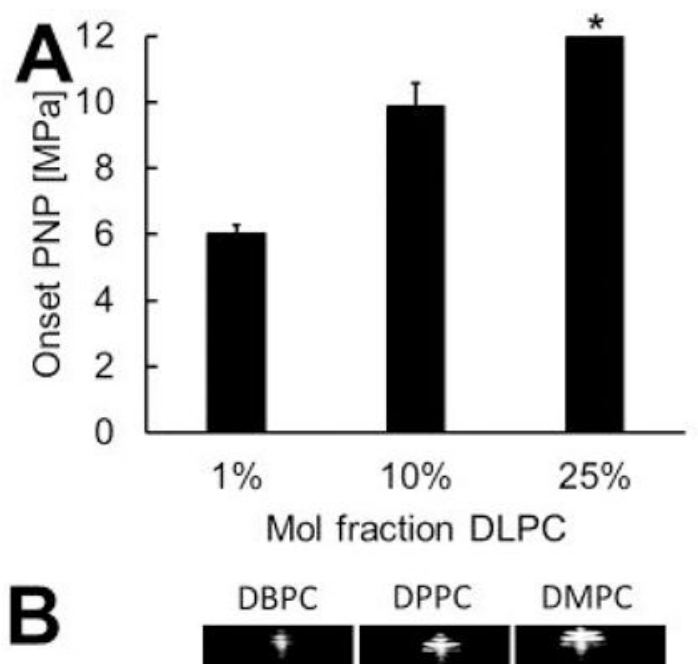

Pure lipid

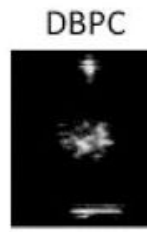

DPPC

DMPC

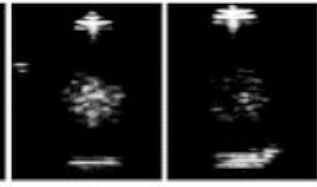

$+50 \% \mathrm{~mol}$

DLPC
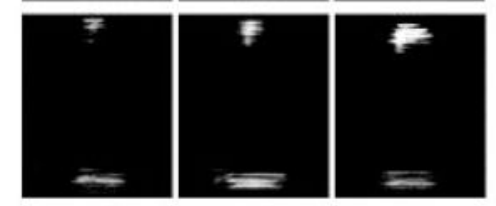

Figure 5.

(A) Onset PNP measurements for DPPC coated hMSNs with varying mole fraction of DLPC. Error bars denote one standard deviation; bars with stars above them denote that no acoustic activity was observed at the maximum PNP of $12 \mathrm{MPa}$. (B) Medical ultrasound images for DBPC, DPPC, and DMPC coated particles with and without $50 \%$ mole fraction DLPC at $10.8 \mathrm{MPa}$ PNP; scale bar refers to approx. $6 \mathrm{~mm}$. For reference, the cross-sectional diameter of the phantom (bound by bright lines at the top and bottom of the image) is 12 $\mathrm{mm}$. 


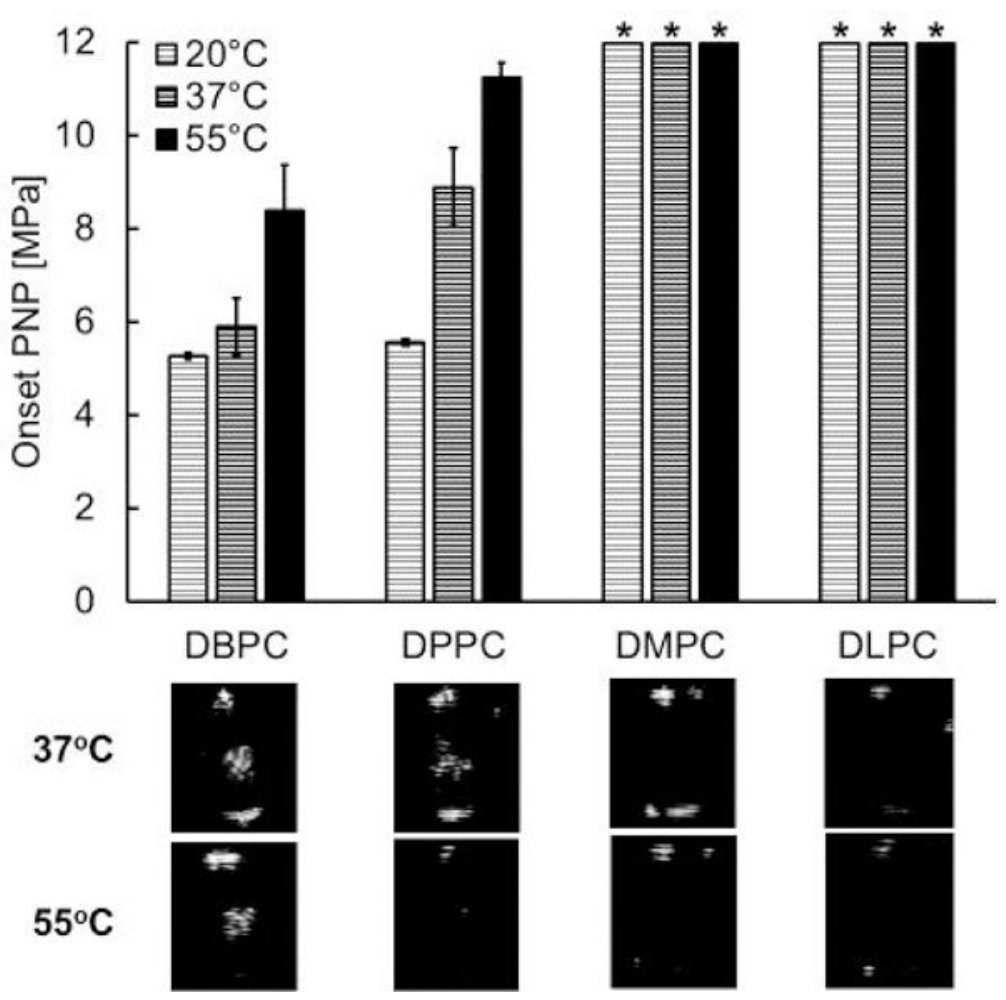

Figure 6.

(Top) PNP measurements of $100 \mu \mathrm{g} / \mathrm{mL}$ P@ hMSNs with decreasing tail length in $90 \%$ bovine plasma at temperatures of 20,37 , and $55^{\circ} \mathrm{C}$. Error bars denote one standard deviation; bars with stars above them denote that no acoustic activity was observed at the maximum PNP of $12 \mathrm{MPa}$. (Bottom) Representative images of medical ultrasound imaging at 10.4 MPa PNP with corresponding phospholipid above. For reference, the cross-sectional diameter of the phantom (bound by bright lines at the top and bottom of the image) is 12 mm. 


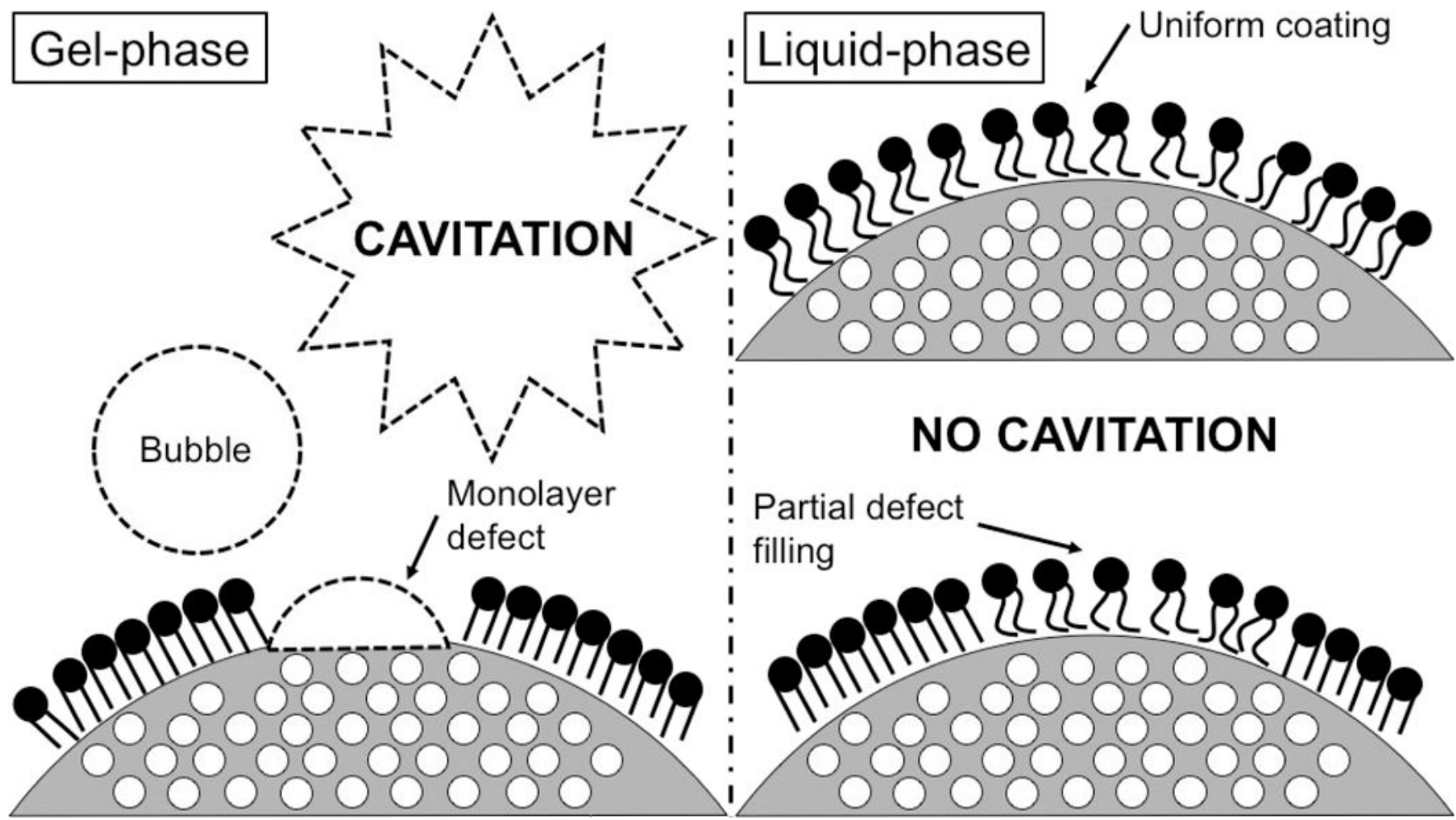

Figure 7.

Schematic showing hypothesis behind acoustic activity as determined by lipid phase. (Left) defects present in the gel-phase phospholipid coating of the hMSN surface give rise to cavities that serve as nucleation sites for the growth and cavitation of bubbles. (Right) When the lipid coating is in the liquid or expanded phase, or when amphiphiles are able to find to these defect sites, the active sites for bubble nucleation are removed from the nanoparticle surface. 